Supporting Information

\title{
Halide Influence on Molecular and Supramolecular Arrangements of Iron Complexes with a 3,5-Bis(2-Pyridyl-1,2,4,6-Thiatriazine Ligand
}

Katie L. M. Harriman, ${ }^{a}$ Irina Kühne, ${ }^{a}$ Alicea A. Leitch, ${ }^{a}$ Ilia Korobkov,${ }^{a}$ Rodolphe Clérac, $, * b, c$ Muralee Murugesu, ${ }^{* a}$ and Jaclyn L. Brusso ${ }^{*, a}$

${ }^{a}$ Department of Chemistry and Centre for Catalysis Research and Innovation, University of Ottawa, Ottawa, Ontario K1N 6N5, Canada.

${ }^{b}$ CNRS, CRPP, UPR 8641, F-33600 Pessac, France.

${ }^{c}$ Univ. Bordeaux, CRPP, UPR 8641, F-33600 Pessac, France.

*Corresponding Authors: J.L. Brusso (E-mail: jbrusso@uottawa.ca; Tel: +1 (613) 5625800 ext 7091); M. Murugesu (E-mail: m.murugesu@uottawa.ca; Tel: +1 (613) 5625800 ext 2733); R. Clérac (E-mail: clerac@ crpp-bordeaux.cnrs.fr; Tel: +33 (0)603517416).

\section{Contents}

Page S2 Experimental Section

Page S2 Electrochemistry

Page S3 Crystal Growth and X-Ray Diffraction

Page S6 Powder X-Ray Diffraction

Page S7 Magnetic Measurements

Page S8 References 
General Procedures and Starting Materials. Ferric chloride (Sigma-Aldrich), ferric bromide (Aldrich), and ferric fluoride (Alfa Aesar) were obtained commercially and used as received unless otherwise stated. $\mathbf{P y}_{2} \mathbf{T T A H}^{1}$ and $\left[\mathbf{F e}\left(\mathbf{P y}_{\mathbf{2}} \mathbf{T T A}\right) \mathbf{C l}_{2}\right]\left(\mathbf{1}^{2}{ }^{2}\right.$ were prepared as outlined in the literature. All solvents were of at least reagent grade. All reactions were carried out under atmospheric conditions. Infrared spectra were recorded on either a Cary 630 or Nicolet Nexus 550 FT-IR spectrometer using Attenuated Total Reflection in the $4000-600 \mathrm{~cm}^{-1}$ range. Elemental analyses were carried out with a Costech EOS 4010 CHNSO analyzer. UV-visible spectra were measured using an Agilent Cary 5000 UV-Vis-NIR spectrophotometer in the range $200-1200 \mathrm{~nm}$. Solution absorption measurements were completed on dichloromethane solutions with standard $10 \mathrm{~mm}$ pathlength cuvettes. Diffuse reflectance measurements were collected with the use of a Praying Mantis ${ }^{\mathrm{TM}}$ accessory by Harrick Scientific. Baseline correction was completed with Spectralon $₫$ reference.

Preparation of [Fe(Py $\left.\left.\mathbf{P y}_{2} \mathbf{T T A}\right) \mathbf{B r}_{2}\right]$ (2). A solution of ferric bromide $(0.188 \mathrm{mmol}, 55.4 \mathrm{mg})$ in

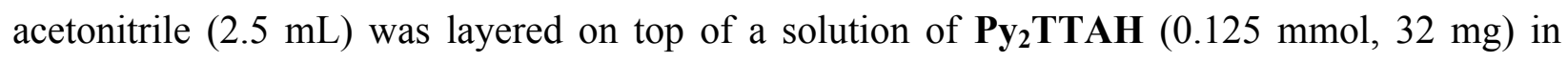
chloroform $(2.5 \mathrm{~mL})$. Green-brown block-like crystals suitable for X-ray diffraction grew from the reaction mixture after 3 days. Yield $=77 \%$. IR (neat, $\mathrm{cm}^{-1}$ ): 3076, 1618, 1604, 1583, 1561, 1474, 1447, 1374, 1295, 1259, 1149, 1082, 1045, 1023, 8117, 799, 765, 754, 711, 683, 652, 598. Calcd for $\mathrm{C}_{12} \mathrm{H}_{8} \mathrm{Br}_{2} \mathrm{FeN}_{5} \mathrm{~S}$ : C, 30.67; H, 1.71; N, 14.90; S, 6.82. Found C, 30.48; H, 1.49; N, 14.70; S, 6.77.

Preparation of $\left[\mathbf{F e}(\boldsymbol{\mu}-\mathbf{F})\left(\mathbf{1 - 0 x o - P y _ { 2 } T T A}\right) \mathbf{F}\right]_{\infty}$ (3). A heterogeneous reaction was prepared with

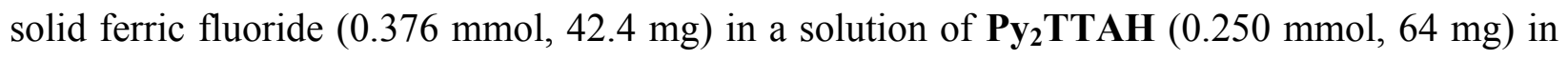
DMF ( $5 \mathrm{~mL})$ and $\mathrm{MeOH}(5 \mathrm{~mL})$. The resulting solution was stirred overnight and filtered to give a dark green solution. The filtrate was placed in a diethyl ether bath at room temperature. Green needle crystals suitable for X-ray diffraction were recovered after several days. Yield $=47 \%$. IR (neat, $\mathrm{cm}^{-1}$ ): 3076, 2161, 1979, 1660, 1618, 1604, 1583, 1562, 1474, 1447, 1373, 1296, 1259, 1172, 1149, 1095, 1082, 1045, 1023, 903, 799, 760, 751, 721, 711, 685, 685, 632, 599, 578, 559, 546. Bulk purity of the sample was determined by powder X-ray diffraction (vide infra).

Electrochemistry. Cyclic voltammetry was performed using a BASi Epsilon potentiostat employing a glass cell and platinum wires for working, counter and pseudo-reference electrodes. The measurements were carried out on dichloromethane solutions (dried by Innovative 
Technology PureSolv solvent system and stored over $4 \AA$ molecular sieves) containing $0.1 \mathrm{M}$ tetrabutylammonium hexafluorophosphate (Aldrich) as supporting electrolyte with a scan rate of $100 \mathrm{mV} / \mathrm{s}$. The experiments were referenced to the $\mathrm{Fc} / \mathrm{Fc}^{+}$redox couple of ferrocene at $+0.48 \mathrm{~V}$ vs. SCE. ${ }^{3}$

Crystal Growth. Crystals of 1 and 2 were grown from a mixture of MeOH/DMF and $\mathrm{CHCl}_{3} / \mathrm{MeCN}$, respectively, at room temperature. Crystals of $\mathbf{3}$ were grown via diethyl ether diffusion into a solution of $\mathrm{MeOH} / \mathrm{DMF}$, at room temperature.

Single Crystal X-ray Diffraction Studies. Data collection results for compounds 2 and $\mathbf{3}$ represent the best data sets obtained in several trials for each sample. The crystals were mounted on thin glass fibers using paraffin oil. Prior to data collection crystals were cooled to $200.15 \mathrm{~K}$. Data were collected on a Bruker AXS KAPPA single crystal diffractometer equipped with a sealed Mo tube source (wavelength $0.71073 \AA$ ) APEX II CCD detector. Raw data collection and processing were performed with APEX II software package from BRUKER AXS. ${ }^{4}$ Diffraction data for 3 were collected with a sequence of $0.3^{\circ} \omega$ scans at 0,120 , and $240^{\circ}$ in $\varphi$. Due to lower unit cell symmetry in order to ensure adequate data redundancy, diffraction data for 2 were collected with a sequence of $0.3^{\circ} \omega$ scans at $0,90,180$ and $270^{\circ}$ in $\varphi$. Initial unit cell parameters were determined from 60 data frames with $0.3^{\circ} \omega$ scan each, collected at the different sections of the Ewald sphere. Semi-empirical absorption corrections based on equivalent reflections were applied. ${ }^{5}$ Systematic absences in the diffraction data and unit-cell parameters were consistent with monoclinic $P 22_{1} / c$ (№14) for 3 and triclinic $P \overline{1}$ (№2) for 2 . Solutions in the centrosymmetric space groups for both compound yielded chemically reasonable and computationally stable results of refinement. The structures were solved by direct methods, completed with difference Fourier synthesis, and refined with full-matrix least-squares procedures based on $F^{2}$.

Diffraction data for the crystal of the complex 3 were collected to $0.75 \AA$ resolution; however, due to the small crystal size and weak diffraction it was discovered that both $R$ (int) and $R$ (sigma) exceed $35 \%$ for the data below $0.90 \AA$ resolution. Based on $R$ (sigma) value data were truncated to $0.85 \AA$ resolution for refinement. Asymmetric unit for this crystallographic model contain one target complex molecule located in the general position. On final stages of 
refinement unsatisfactory thermal parameters as well as analysis of variance for employed reflections suggested the presence of pseudo-merohedral twinning. Data was processed through TwinRotMat subroutine of PLATON software package. ${ }^{6}$ Determined domains rotation matrix was introduced in the refinement with TWIN command. Twinning domain ratio (BASF) was refined to 0.23516 . In order to obtain acceptable values for atomic thermal motion coefficients set of thermal motion parameters constrains (SIMU, RIGU) was employed for all the atoms in the structural mode.

Diffraction data for the crystal of the complex 2 were collected to $0.75 \AA$ resolution, however due to small crystal size and weak diffraction it was discovered that both $R$ (int) and $R$ (sigma) exceed $35 \%$ for the data below $1.00 \AA$ resolution. Based on $R$ (sigma) value data were truncated to $0.95 \AA$ resolution for refinement. Asymmetric unit for this crystallographic model contain one target complex molecule located in the general position. Refinement results for the compound 2 suggested presence of several non-merohedrally twinned domains. Careful examination of the original data frames and reciprocal space diffraction pictures confirmed the initial twining assumption. In order to find independent orientation matrices 1553 reflections were collected from 4 sets of 50 frames each in the different sections of the Ewald sphere. Collected reflection data were processed with CELL_NOW software ${ }^{7}$ and produced three independent orientation matrices. Data set was re-integrated with three obtained matrices, treated for twinning absorption corrections and consecutive model refinement was performed using HKLF5 formatted reflection data file. Twinning domain ratio coefficients (BASF) was refined to 0.45477 and 0.03967 . In order to obtain acceptable values for atomic thermal motion coefficients set of thermal motion parameters constrains (SIMU, RIGU) was employed for all the atoms in the structural mode. The highest residual peaks in the difference electron density map are located near the heavy atoms $(0.96 \AA$ from $\mathrm{Br}$ for the highest peak) and most probably are a consequence of twinning/Fourier truncation.

For both compounds all non-hydrogen atoms were refined with anisotropic thermal motion approximation. All hydrogen atom positions were calculated based on the geometry of related non-hydrogen atoms. All hydrogen atoms were treated as idealized contributions during the refinement. All scattering factors are contained in several versions of the SHELXTL program library, with the latest version used being v.6.12. ${ }^{8}$ 
Table S1. Crystallographic data and selected data collection parameters.

\begin{tabular}{|c|c|c|}
\hline Compound & {$\left[\mathrm{Fe}\left(\mathrm{Py}_{2} \mathrm{TTA}_{\mathrm{A}}\right) \mathrm{Br}_{2}\right]$} & {$\left[\mathrm{Fe}(\mu-\mathrm{F})\left(1-0 \times 0-P y_{2} \text { TTA }\right) \mathrm{F}\right]_{\infty}$} \\
\hline Empirical formula & $\mathrm{C}_{12} \mathrm{H}_{8} \mathrm{Br}_{2} \mathrm{FeN}_{5} \mathrm{~S}$ & $\mathrm{C}_{12} \mathrm{H}_{8} \mathrm{~F}_{2} \mathrm{FeN}_{5} \mathrm{OS}$ \\
\hline Formula weight & 469.96 & 364.14 \\
\hline Crystal size, $\mathrm{mm}$ & $0.15 \times 0.10 \times 0.09$ & $0.12 \times 0.03 \times 0.01$ \\
\hline Crystal system & triclinic & monoclinic \\
\hline Space group & $P-1$ & $\boldsymbol{P} 2_{1} / c$ \\
\hline$a, \AA$ & $7.609(3)$ & $13.8328(13)$ \\
\hline$b, \AA$ & $8.283(3)$ & $13.7235(14)$ \\
\hline$c, \AA$ & $13.493(7)$ & $7.5214(7)$ \\
\hline$\alpha,{ }^{\circ}$ & $75.61(2)$ & 90 \\
\hline$\beta,^{\circ}$ & $89.52(3)$ & $104.868(5)$ \\
\hline$\gamma,^{\circ}$ & $63.74(2)$ & 90 \\
\hline Volume, $\AA^{3}$ & $733.7(6)$ & $1380.0(2)$ \\
\hline$Z$ & 2 & 4 \\
\hline Calculated density, $\mathrm{Mg} / \mathrm{m}^{3}$ & 2.127 & 1.753 \\
\hline Absorption coefficient, $\mathrm{mm}^{-1}$ & 6.614 & 1.275 \\
\hline$T(\mathrm{~K})$ & $200(2)$ & $200(2)$ \\
\hline $\mathrm{F}(000)$ & 454 & 732 \\
\hline$\Theta$ range for data collection, $^{\circ}$ & 2.85 to 24.76 & 1.48 to 26.36 \\
\hline Limiting indices & $h= \pm 8, k= \pm 9, l= \pm 15$ & $h= \pm 17, k= \pm 17, l= \pm 9$ \\
\hline Reflections collected / unique & 4085 / 2207 & $16032 / 2704$ \\
\hline$R($ int $)$ & 0.1008 & 0.0790 \\
\hline Completeness to $\Theta=28.32, \%$ & 90.6 & 99.4 \\
\hline Max. and min. transmission & 0.7451 and 0.3399 & 0.6028 and 0.7454 \\
\hline Data / restraints / parameters & $2207 / 327$ / 192 & 2704 / 348 / 200 \\
\hline Goodness-of-fit on $F^{2}$ & 1.090 & 1.088 \\
\hline Final $R$ indices $[I>2 \sigma(I)]$ & $R_{1}=0.1171, w R_{2}=02643$ & $R_{1}=0.0780, w R_{2}=0.1877$ \\
\hline$R$ indices (all data) & $R_{1}=0.1721, w R_{2}=0.2985$ & $R_{1}=0.1327, w R_{2}=0.2158$ \\
\hline Largest diff. peak/hole, e $\cdot \AA^{-3}$ & $1.676 /-1.908$ & $2.641 /-0.679$ \\
\hline
\end{tabular}



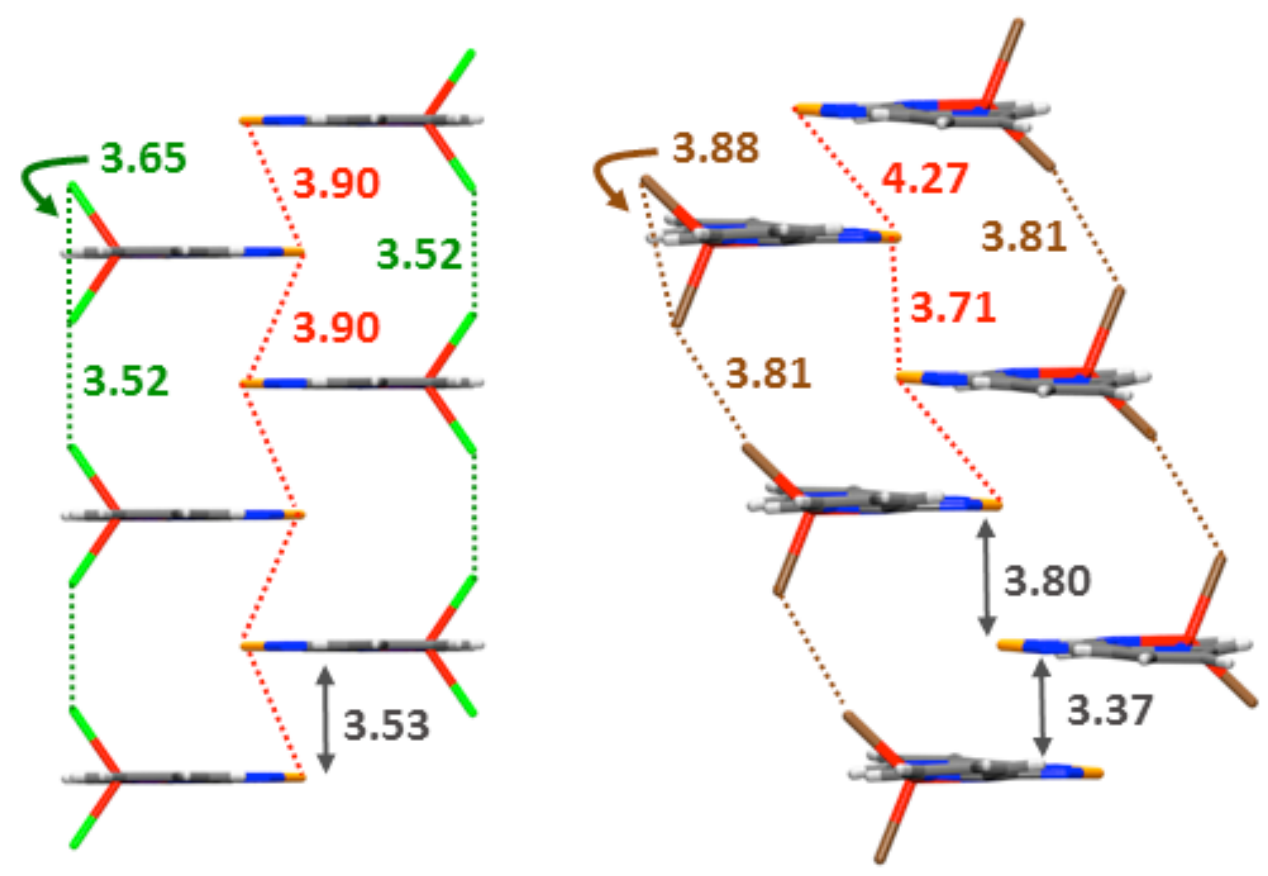

Figure S1. Packing diagram exhibiting a ladder-like arrangement of $\left[\mathbf{F e}\left(\mathbf{P y}_{2} \mathbf{T T A}\right) \mathbf{C l}_{2}\right]$ (left) and $\left[\mathbf{F e}\left(\mathbf{P y}_{2} \mathbf{T T A}\right) \mathbf{B r}_{2}\right]$ (left) in the solid state. Selected distances are shown for intramolecular and intermolecular interactions.

Powder X-ray Diffraction (PXRD) of $\left[\mathrm{Fe}(\boldsymbol{\mu} \text {-F)(1-oxo-Py } 2 \text { TTA)F }]_{\infty}(3)\right.$. The X-ray powder diffraction analysis of a bulk sample of $\mathbf{3}$ was carried out using a Rigaku Ultima IV X-ray powder diffractometer equipped with a $\mathrm{Cu}-\mathrm{K} \alpha$ radiation source $(\lambda=1.541836 \AA)$, and a graphite monochromator. The parallel beam mode was used to collect the data. PXRD pattern was consistent in $2 \theta$ values with the generated pattern from the single crystal X-ray diffraction data (XRD), with slight discrepancies in some intensities of peaks attributed to preferred orientation due to limited sample size. 


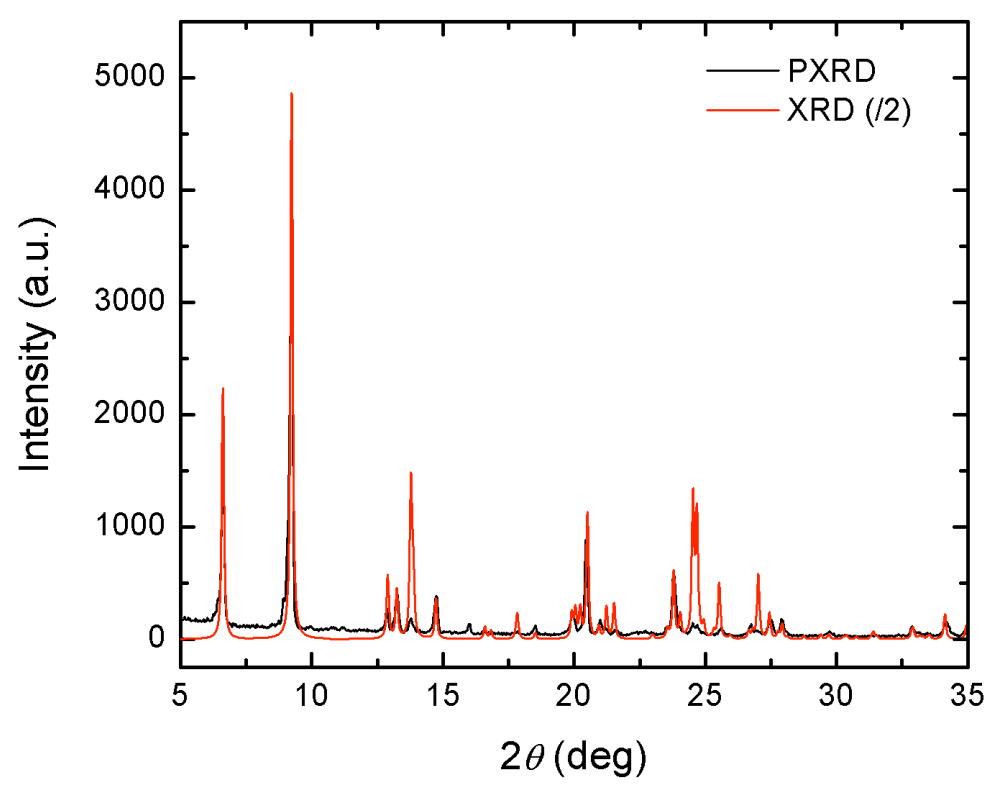

Figure S2. Powder X-ray diffraction of $\left[\mathbf{F e}(\boldsymbol{\mu}-\mathbf{F})\left(\mathbf{1 - 0 x 0 - P y _ { 2 } T T A ) F}\right]_{\infty}\right.$, experimentally derived pattern (black) and calculated pattern from XRD data (red) in the 5-35 $2 \theta$ region.

Magnetic Measurements. The magnetic susceptibility measurements were obtained using a Quantum Design SQUID magnetometer MPMS-XL7 operating between 1.85 and $300 \mathrm{~K}$ with a dc field between $-7 \mathrm{~T}$ and $7 \mathrm{~T}$. For alternating-current (ac) susceptibility measurements, an oscillating ac field of 3 Oe with a frequency between 1 and $1500 \mathrm{~Hz}$ were employed in zero-dc field. Magnetic measurements were performed on a polycrystalline samples of $18.2 \mathrm{mg}, 13.3 \mathrm{mg}$,

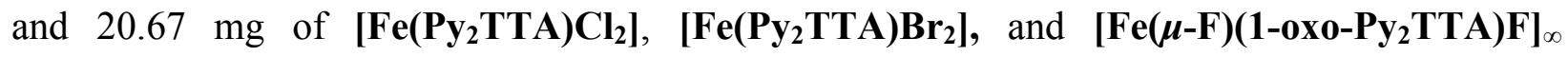
respectively. Samples were transferred in a polyethylene bag $(3 \times 0.5 \times 0.02 \mathrm{~cm}$; typically of 20 $30 \mathrm{mg}$ ). Prior to the experiments, the field-dependent magnetization was measured at $100 \mathrm{~K}$ in order to detect the presence of any bulk ferromagnetic impurities. The sample appeared to be free of any significant ferromagnetic impurities. The magnetic data were corrected for the sample holder and the diamagnetic contribution. 


\section{References}

1. Leitch, A. A.; Korobkov, I.; Assoud, A.; Brusso, J. L., Chemical communications (Cambridge, England) 2014, 50 (38), 4934-4936.

2. Harriman, K. L. M.; Leitch, A. A.; Stoian, S. A.; Habib, F.; Kneebone, J. L.; Gorelsky, S. I.; Korobkov, I.; Desgreniers, S.; Neidig, M. L.; Murugesu, M.; Brusso, J. L., Dalton Transactions 2015, 44, 10516-10523.

3. Boere, R. T.; Moock, K. H.; Parvez, M., Zeitschrift Fur Anorganische Und Allgemeine Chemie 1994, 620 (9), 1589-1598.

4. $\quad$ APEX Software Suite v.2012; Bruker AXS: Madison, WI., 2005.

5. Blessing, R. H., Acta Crystallographica Section A 1995, 51, 33-38.

6. Spek, A. L., Acta Crystallographica Section D-Biological Crystallography 2009, 65, 148155.

7. Sheldrick, G. M., Cell_Now, Bruker-AXS. Madison, WI, 2004.

8. Sheldrick, G. M., Acta Crystallographica Section A 2008, 64, 112-122. 